\title{
Robot modeling and simulation analysis of micro-gravity conditions
}

\author{
Qiaolong Yang ${ }^{1, a}$, Hongwei Liu' \\ ${ }^{1}$ Beijing Institute of Spacecraft System Engineering, Beijing 100094, China \\ ${ }^{2}$ School of Aerospace Science, Beijing Institute of Technology, Beijing 100081, China \\ a37640647@qq.com, b1132520084@qq.com
}

Keywords: Space Manipulator, Kinematics, Dynamics, Compound Force-field

\begin{abstract}
With the development of space technology, the space manipulator is widely used in space. Before launching into space to practical work, the manipulator needs to finish a large of number of experiments on the ground. In this article, we study on the mechanical arm to finding out the mechanical properties in the ground simulation experimental system and put forward the ground microgravity experiment system with a balance beam structure. Based on the D-H method, the mathematical model of the mechanical arm is established. And it's obvious that the microgravity experiment system scheme is feasible to solve the kinematics equation of manipulator. Based on the Lagrange method, the dynamics model of manipulator is established. Finally a dynamic simulation of mechanical arm based on MATLAB and ADAMS has demonstrated effective.
\end{abstract}

\section{Introduction}

The analysis of the mechanical arm under the complex force field is mainly divided into kinematics and dynamics research. The foundation of dynamic modeling manipulator is kinematics analysis, which can be divided into two parts-- positive and inverse kinematics. Although there are many methods of describing the motion parameters of the manipulator, the most classic kinematic model is the D-H model given by Danevit and Hartenberg [1,2].

The study of the mechanical arm dynamics is concentrated on the relationship between kinematic variables such as angle, angular velocity and angular acceleration, and the force and moment. And it's also two aspects of forward and inverse problems. There are many methods to establish the dynamic model, such as Newton-Euler's method, Lagrange method, Kane method, the Gaussian method, Roberon-Wittenburg method [3-5].

In the past few decades, the dynamic modeling of space manipulator has attracted many scholars' attention and several methods for describing the dynamics of a space manipulator have been proposed [6]. For example, Vafa and Dubowsky presents the concept of virtual robotic arm [7]. Virtual fixed base space manipulator arm with the original has the same dynamics and kinematics characteristics, also known as dynamic equivalence manipulator [8], Dynamic modeling has been used to implement equivalent manipulator adaptive control for free-floating space Manipulator.

However the study of manipulator dynamics are done at the zero -gravity environment in space, little attention is paid to the ground test environment [9], which is also called the complex stress field. So this paper concentrate on the analysis of the kinematics and dynamics property under the ground test environment.

The rest of the paper is organized as follows. Section II presents an overview of the micro-gravity system, the modeling process of manipulator kinematics and the analysis of the manipulator dynamics. Experiments and results in the ADAMS and MATLAB are given in Section III. Finally, the paper is concluded in Section IV.

\section{Material and Methods}

The component of the microgravity simulation system. In this paper, gravity simulation system based on the suspension method is used to provide the zero-gravity environment, and the structure is 
shown in the Fig. 1. The manipulator consists of two arms and each arm constitutes a gravity compensation subsystem. The way to realize microgravity is that moving the suspension point guarantee the moving of the center of manipulator arm.

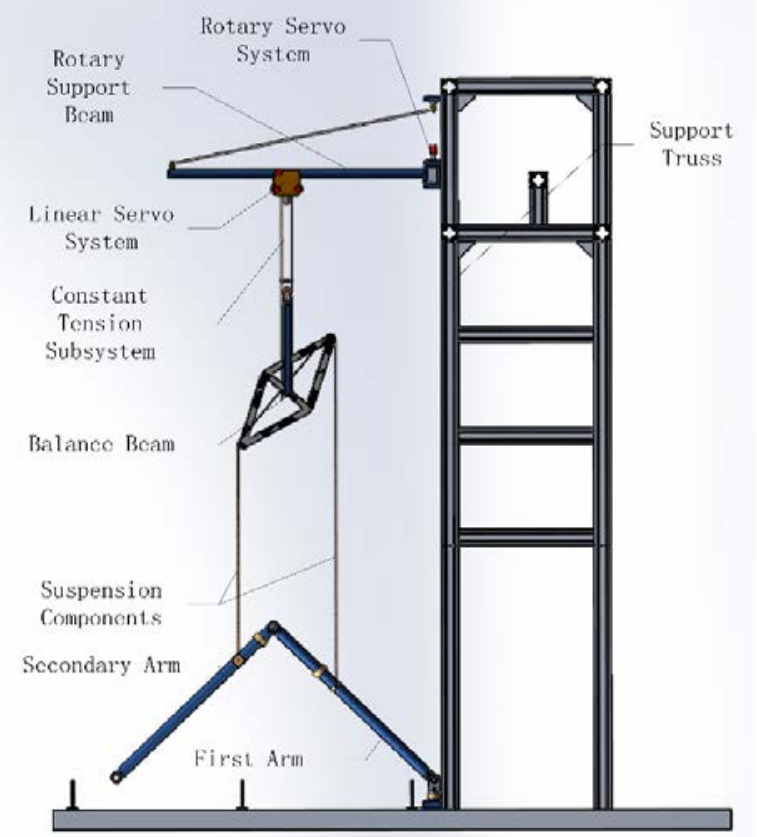

Fig. 1 schematic diagram of manipulator ground simulation system

First, the arm motion data is acquired and send to the measurement and control subsystem to analyze the motion, then motion control instructions is send to the rotation servo subsystem and two Linear servo subsystems, and each subsystem's motion controllers drive the motor to realize the control loop. Suspension point is set in the barycentric position of the arm. The upward pull provided by the hanging devices is equal to the gravity of the mechanical arm, so the effect of the gravity unloading is good. In this paper, the manipulator consists of three joints and a terminal sampler, and the root joint is fixed to the base. So the uploading scheme is searching the center of each arm and fixes the suspending position above the center.

Manipulator kinematics modeling. In order to describe the translation and rotation relationship between adjacent levers, Denavit and Hartenberg proposed a common approach in 1955 . The manipulator D-H coordinate system is established in the Fig. 2. Where the $O_{0} X_{0} Y_{0} Z_{0}$ coordinate system is fixed on the base of the manipulator. Coordinate origin is the intersection of the rotary joint axis of the robot arm and mounting base and Y-axis direction of each joint point coordinates determined by the right-hand rule. According to the characteristics and movement of the suspension arm, Table 1 gives the structural parameters and motion parameters of each rod, namely the D-H parameters.

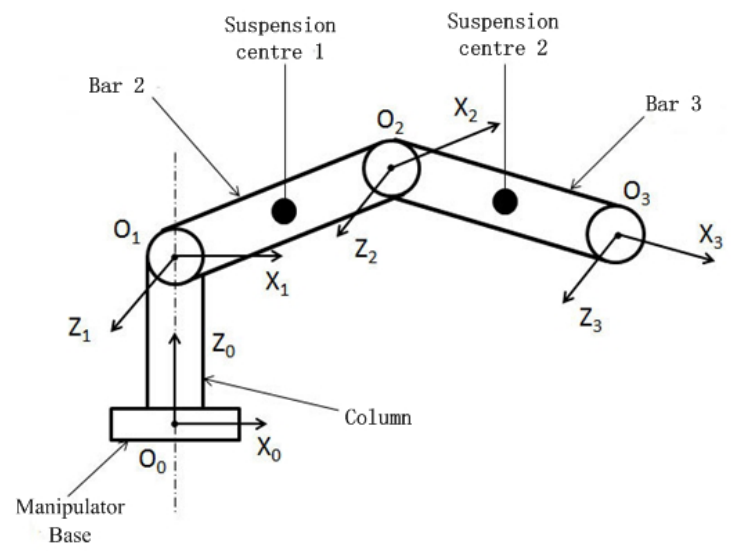

Fig. 2 D-H linkage arm coordinate system 


\begin{tabular}{cccccc} 
Bar number & Bar length & $\begin{array}{c}\text { Linkage corner } \\
\alpha_{i-1}\end{array}$ & $\begin{array}{c}\text { Linkage } \\
\text { offset } d_{i}\end{array}$ & $\begin{array}{c}\text { Variable } \\
\theta_{i}\end{array}$ & Linkage $a_{i}$ \\
\hline 1 & $l_{1}$ & $90^{\circ}$ & $l_{1}$ & $\theta_{1}$ & 0 \\
2 & $l_{2}$ & 0 & 0 & $\theta_{2}$ & $l_{2}$ \\
3 & $l_{3}$ & 0 & $d_{3}$ & $\theta_{3}$ & $l_{3}$ \\
\hline
\end{tabular}

According to the D-H parameters of the manipulator, the transformation matrix of the base to the end effector is as follows:

$$
\begin{aligned}
{ }^{0} T_{1} & =\left[\begin{array}{cccc}
\cos \theta_{1} & 0 & \sin \theta_{1} & 0 \\
\sin \theta_{1} & 0 & -\cos \theta_{1} & 0 \\
0 & 1 & 0 & l_{1} \\
0 & 0 & 0 & 1
\end{array}\right] \\
{ }^{1} T_{2} & =\left[\begin{array}{cccc}
\cos \theta_{2} & -\sin \theta_{2} & 0 & l_{2} \cos \theta_{2} \\
\sin \theta_{2} & \cos \theta_{2} & 0 & l_{2} \sin \theta_{2} \\
0 & 0 & 1 & 0 \\
0 & 0 & 0 & 1
\end{array}\right] \\
{ }^{2} T_{3} & =\left[\begin{array}{cccc}
\cos \theta_{3} & -\sin \theta_{3} & 0 & l_{3} \cos \theta_{3} \\
\sin \theta_{3} & \cos \theta_{3} & 0 & l_{3} \sin \theta_{3} \\
0 & 0 & 1 & d_{3} \\
0 & 0 & 0 & 1
\end{array}\right]
\end{aligned}
$$

Where ${ }^{0} T_{1}$ is the transformation matrix of bar 1 to the base, ${ }^{1} T_{2}$ is the transformation matrix of bar 2 to bar 1 , and ${ }^{2} T_{3}$ is the transformation matrix of bar 3 to bar 2 .

By the matrix multiplication, we can get the transformation matrix of each link coordinate system relative to the base:

$$
\begin{aligned}
& { }^{0} T_{2}={ }^{0} T_{1}{ }^{1} T_{2} \\
& { }^{1} T_{3}={ }^{1} T_{2}{ }^{2} T_{3} \\
& { }^{0} T_{3}={ }^{0} T_{1}{ }^{1} T_{2}{ }^{2} T_{3}
\end{aligned}
$$

Where ${ }^{0} T_{3}$ is the terminal's transform matrix.

Therefore, the attitude of the end connecting rod coordinate system relatived to the base coordinate system is shown below:

$$
\begin{aligned}
& n_{x}=c_{1} C_{2} C_{3}-c_{1} S_{2} S_{3} \quad o_{x}=-C_{1} C_{2} S_{3}-c_{1} C_{3} S_{2} \\
& n_{y}=C_{2} C_{3} S_{1}-S_{1} S_{2} S_{3} \quad o_{y}=-C_{2} S_{1} S_{3}-C_{3} S_{1} S_{2} \\
& n_{z}=c_{2} S_{3}+c_{3} S_{2} \quad O_{z}=c_{2} C_{3}-S_{2} S_{3} \\
& a_{x}=s_{1} \quad p_{x}=d_{3} s_{1}+l_{2} c_{1} c_{2}-c_{1} l_{3} s_{2} s_{3}+c_{1} c_{2} c_{3} l_{3} \\
& a_{y}=-c_{1} \quad p_{y}=c_{2} l_{2} s_{1}-c_{1} d_{3}+c_{2} c_{3} l_{3} s_{1}-l_{3} s_{1} s_{2} s_{3} \\
& a_{z}=0 \quad p_{z}=l_{1}+l_{2} s_{2}+c_{2} l_{3} s_{3}+c_{3} l_{3} s_{2}
\end{aligned}
$$

Eq. 6 represents the end of the link coordinate system relative to the position and orientation of the base coordinate system. 
The solution of mechanical arm dynamics under compound force field. Kinetic analysis is the basis of machine design, performance analysis and control of balance arm. It has two problems of different directions: 1, known driving force or moment of each joint, solve their location, velocity and acceleration. It is forward dynamies analysis. 2, known location, velocity and acceleration of each joint, solve its required driving force or moment. It is dynamic inverse problem. The direct problem is mainly used for motion simulation while inverse problem is to control movement of the balance arm effectively to achieve expectant track and achieve good dynamic performance [10].

Lagrange function $L$ is defined as the difference of kinetic energy $K$ and potential energy $P$ of system. That is,

$$
L=K-P
$$

Among them, $K$ and $P$ can be expressed by any convenient coordinate system.

Under compound force field, we simplify the sampling apparatus mechanical arm model and unload device added mass is shown as the barycentric position of the connecting rod quality as Fig. 3. In the graph, $m_{1}, m_{2}$ and $m_{3}$ are respectively the quality of upright, level 1 arm and level 2 arm and they are shown as the quality of the barycentric position of the connecting rod points. $l_{1}, l_{2}$ and $l_{3}$ are respectively length of upright, level 1 arm and level $2 \mathrm{arm} ; \theta_{1}, \theta_{2}$ and $\theta_{3}$ are generalized coordinates; is acceleration of gravity. The paper analyses mechanical arm by Lagrangian method.

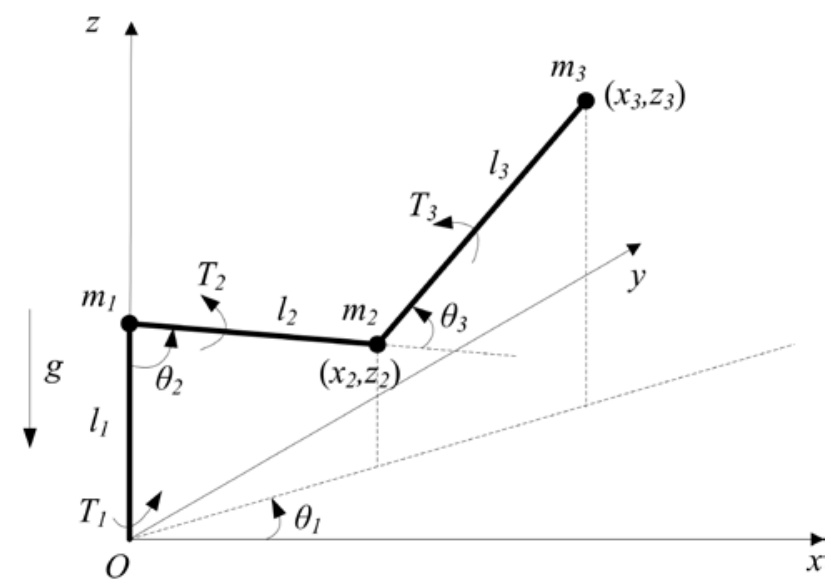

Fig. 3 Simplified diagram of mechanical arm (compound force field)

We can reach mechanical arm's total kinetic energy $K_{a}$ and total potential energy $P_{a}$ under the compound force field are shown below:

$$
\begin{aligned}
K_{a}= & K_{1}+K_{2}+K_{3}=\frac{1}{4} m_{1} R_{1}^{2} \dot{\theta}_{1}^{2}+\frac{1}{8}\left(m_{2}+M_{2}\right) l_{2}^{2} \sin ^{2} \theta_{2} \dot{\theta}_{1}^{2}+\frac{1}{8}\left(m_{2}+M_{2}\right) l_{2}^{2} \dot{\theta}_{2}^{2} \\
+ & \frac{1}{2}\left(m_{3}+M_{3}\right)\left[l_{2} \sin \theta_{2}+\frac{1}{2} l_{3} \sin \left(\theta_{2}+\theta_{3}\right)\right]^{2} \dot{\theta}_{1}^{2}+\frac{1}{2}\left(m_{3}+M_{3}\right) l_{2}^{2} \dot{\theta}_{2}^{2} \\
& +\frac{1}{8}\left(m_{3}+M_{3}\right) l_{3}^{2}\left(\dot{\theta}_{2}+\dot{\theta}_{3}\right)^{2}+\frac{1}{2}\left(m_{3}+M_{3}\right) l_{2} l_{3} \cos \theta_{3}\left(\dot{\theta}_{2}^{2}+\dot{\theta}_{2} \dot{\theta}_{3}\right) \\
P_{a}= & P_{1}+P_{2}+P_{3} \\
= & \frac{1}{2} m_{1} a l_{1}+\left(m_{2}+M_{2}\right) a\left(l_{1}-\frac{1}{2} l_{2} \cos \theta_{2}\right) \\
& +\left(m_{3}+M_{3}\right) a l_{1}-\left(m_{3}+M_{3}\right) a l_{2} \cos \theta_{2}-\frac{1}{2}\left(m_{3}+M_{3}\right) a l_{3} \cos \left(\theta_{2}+\theta_{3}\right)
\end{aligned}
$$

Then we can obtain Lagrange function $L_{a}$ of analog system under the compound force field:

$$
L_{a}=K_{a}-P_{a}
$$

Next, we can acquire size of each Joint torque $T_{a 1}, T_{a 2}$ and $T_{a 3}$ by taking partial derivative and differential coefficient to $L_{a}$ then taking them to Eq. 19 . 


$$
\begin{aligned}
& T_{a 1}=\frac{d}{d t} \frac{\partial L}{\partial \dot{\theta}_{1}}-\frac{\partial L}{\partial \theta_{1}} \\
& T_{a 2}=\frac{d}{d t} \frac{\partial L}{\partial \dot{\theta}_{2}}-\frac{\partial L}{\partial \theta_{2}} \\
& T_{a 3}=\frac{d}{d t} \frac{\partial L}{\partial \dot{\theta}_{3}}-\frac{\partial L}{\partial \theta_{3}}
\end{aligned}
$$

So far, we obtain the applied moment imposed on mechanical arm's each joint driver under the compound force field.

\section{Results and Discussion}

The combined-simulation to manipulator dynamics in the paper is accomplished by MATLAB and ADAMS and the mechanical arm's motor process of dynamics simulation under the compound force field is set as uniformly accelerated motion. The shoulder jaw joint angle $\theta_{1}$ 's accelerated speed is $\ddot{\theta}_{1}=0.5^{\circ} / \mathrm{s}^{2}$; Shoulder pitch joint Angle $\theta_{2}$ 's accelerated speed is $\ddot{\theta}_{2}=-0.5^{\circ} / \mathrm{s}^{2}$; The elbow pitch joint Angle $\theta_{3}$ 's accelerated speed is $\ddot{\theta}_{3}=1^{\circ} / \mathrm{s}^{2}$.

The compound force field environment is established in ADAMS: First of all, Introduce gravity field environment, set additional quality parameters and make added mass of unloading device is equal to the All levels of the mechanical arm's quality themselves. Set unloading strength parameters, make it equal to gravity of mechanical arm's additional unloading device and its direction contrary to gravitational field. Considering unloading system error, we introduce uninstall error $\delta=-0.5 \%$. The minus presents that imitation experiment system has unloading problem.

Under the compound force field motion state, each joint's applied moment graphs are shown as Fig. 4-Fig. 6.
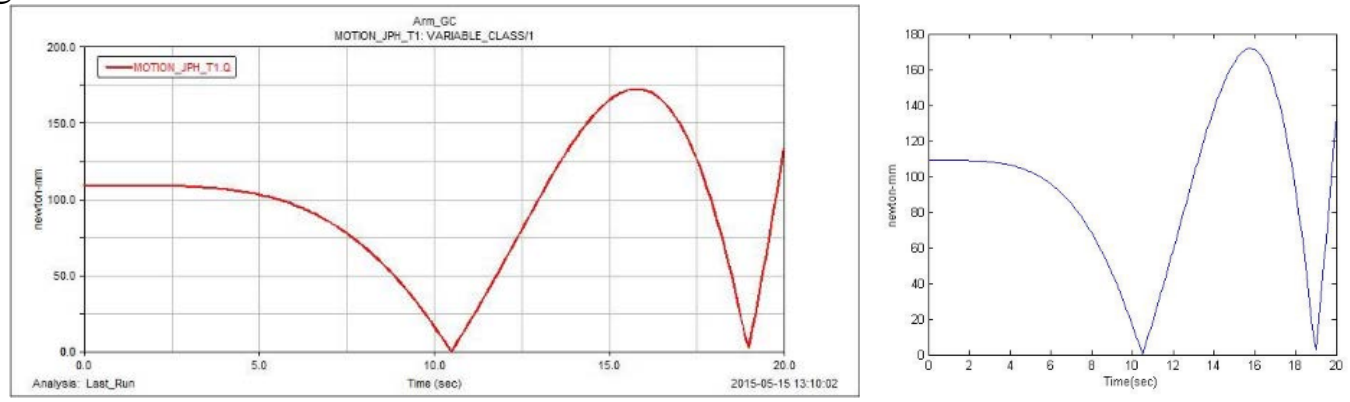

1) ADAMS simulation result $\quad$ 2) Theoretical calculation results

Fig. 4 Torque of the shoulder yaw joint in the compound force field
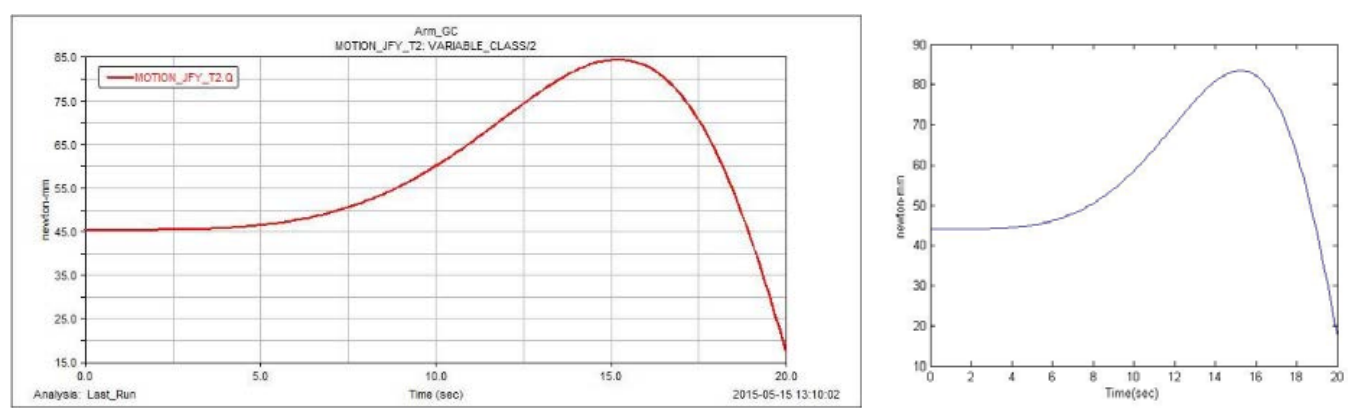

1)ADAMS simulation result $\quad$ 2)Theoretical calculation results

Fig. 5 Torque of the shoulder pitch joint in the compound force field 

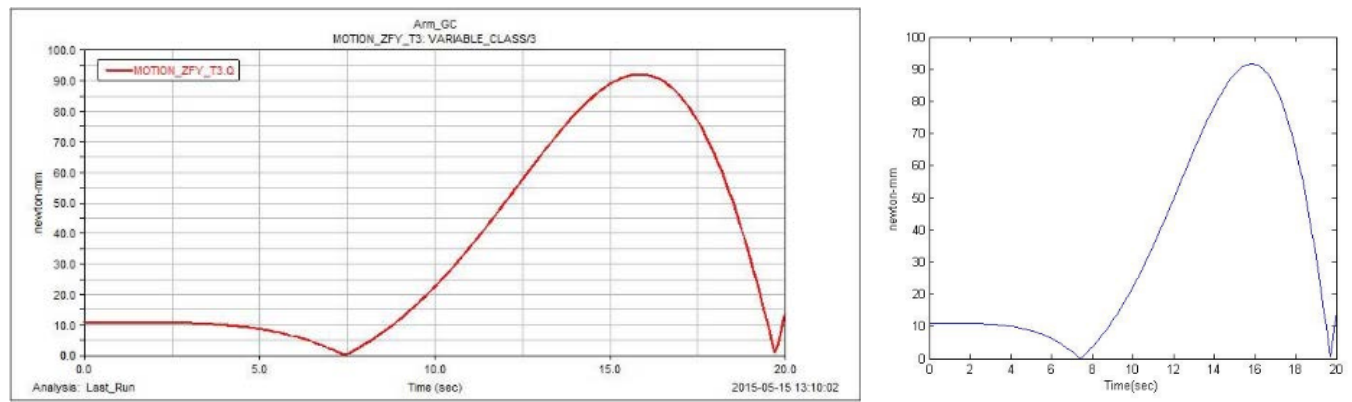

1)ADAMS simulation result

2)Theoretical calculation results

Fig. 6 Torque of the elbow yaw joint in the compound force field

From the Fig. 4-Fig. 6, we can figure out that mechanical arm each joint's moment graph's variation trend by simulation result under the compound force field is same as by mathematical model. The moment size's data error is within $1 \%$ and error value is within tolerance interval.

\section{Conclusion}

According to the demand of space robotic manipulator, the paper designs ground microgravity testing system unloading plan. We proceed kinematical modeling to mechanical arm by D-H method and reach sampling apparatus's spatial position and attitude matrix in the tail end of mechanical arm. In the same time, we proceed dynamics modeling to mechanical arm by Lagrangian method and reach mechanical arm's kinetic model under the compound force field. Finally, we proceed joint simulation by ADAMS and MATLAB and verify effectiveness of the model.

\section{References}

[1] J. Denavit and R. S. Hartenberg. Trans.of the Asme.journal of Applied Mechanics, 1955, 22: 215.

[2] W. Feng, W. M. Yi , F. W. Meng, X. P. Shen and X. D. Song. Advanced Materials Research, 2014, 940: 321.

[3] K. S. Anderson and J. H. Critchley. Multibody System Dynamics, 2003, 9(2): 185.

[4] L. Zhen , M. Jun and C. Hong-yue. Journal of Jilin University(Information Science Edition), 2013, 31(3): 290.

[5] C. Genliang , W. Hao , L. Xinmin and L. Zhongqin. JOURNAL OF MECHANICAL ENGINEERING, 2009, 45(7): 41.

[6] J. Li. Research on ground microgravity compensation experiment system based on the suspension spring: University Of Science And Technology Of China, 2014.

[7] Z. Vafa and S. Dubowsky. On The Dynamics Of Manipulators In Space Using The Virtual Manipulator Approach, 1987. 579.

[8] L. BIN , L. LIANGDONG , L. I. GENGTIAN and X. U. YANGSHENG. ACTA AUTOMATICA SINICA, 1998, 24(6): 761.

[9] D. R. Isenberg , S. Baek , M. Caddell , M. Mueller , D. Hill and Y. P. Kakad. A Method of Gravity Offloading with a SCARA Manipulator, 2011. 165.

[10] Y. Chen. Design and dynamic characteristic research of month soil sampling mechanical arm: Harbin Institute of Technology, 2009. 